\section{Coocorrência de fatores de risco obesogênicos em adolescentes brasileiros: o papel das características sociodemográficas e da presença parental}

\section{Cooccurrence of obesogenic risk factors in Brazilian adolescents: the role of sociodemographic characteristics and parental presence}

\section{Coocurrencia de factores de riesgo obesogénicos en adolescentes brasileños: el papel de las características sociodemográficas y presencia parental}

Otaviana Cardoso Chaves 1 Gustavo Velasquez-Melendez 1 Dário Alves da Silva Costa 1 Roseli Gomes de Andrade 1 Waleska Teixeira Caiaffa ${ }^{1}$

doi: 10.1590/0102-311X00013120

\section{Resumo}

O objetivo do estudo foi estimar a prevalência da coocorrência de fatores de risco obesogênicos em adolescentes brasileiros e características sociodemográficas e familiares associadas. É um estudo transversal, com dados da Pesquisa Nacional de Saúde do Escolar, $2009(n=53.274)$. A variável resposta (coocorrência de fatores de risco obesogênicos) variou de zero a cinco e foi obtida com base no somatório dos comportamentos de risco: consumo diário de refrigerantes; consumo diário de guloseimas; consumo insuficiente de frutas; consumo insuficiente de hortaliças e atividade física insuficiente. As variáveis explicativas foram sexo, idade, cor da pele, região brasileira, escore de bens e serviços do domicílio, escolaridade materna, tipo de escola, composição familiar, monitoramento parental e realização de refeições principais com os responsáveis. Utilizou-se o diagrama de Venn para a análise exploratória e a regressão logística ordinal com modelo de odds proporcionais parciais para análise multivariada. Verificou-se maior chance de ocorrência simultânea dos fatores nos adolescentes do sexo feminino, naqueles que tinham menor monitoramento parental e que realizavam menor número de refeições com os responsáveis. Em contraste, adolescentes que residiam em regiões mais desenvolvidas do país e aqueles cujas mães possuíam maior escolaridade apresentaram menor chance de coocorrência de fatores de risco obesogênicos. Ações que estimulem mais envolvimento e supervisão familiar no comportamento alimentar podem promover impacto positivo na prevenção da obesidade em adolescentes brasileiros, principalmente em regiões menos desenvolvidas e em lares com mães de menor escolaridade.

Obesidade; Adolescente; Consumo de Alimentos; Fatores de Risco

\author{
Correspondência \\ O. C. Chaves \\ Rua Capitão Virgílio Marques 180, Campos Altos, MG \\ 38970-000, Brasil. \\ otavianac@yahoo.com.br \\ 1 Universidade Federal de Minas Gerais, Belo Horizonte, Brasil.
}




\section{Introdução}

A obesidade é considerada, globalmente, um problema de saúde pública que vem aumentando em todas as idades, tanto em países desenvolvidos quanto naqueles em desenvolvimento 1,2. Estudos epidemiológicos têm demonstrado a relação de vários fatores de risco modificáveis com essa morbidade. Em adolescentes, o elevado consumo de refrigerantes 3 e de outros produtos ultraprocessados, com elevada densidade energética e baixo teor de nutrientes 4 , tem sido associado ao excesso de peso.

Embora o baixo consumo de frutas e hortaliças 5 e o menor nível de atividade física 6 se mostre associado de forma menos evidente com as alterações do estado nutricional, estas associações são plausíveis. Frutas e hortaliças geralmente apresentam baixa densidade energética, elevado teor de água e uma quantidade considerável de fibras 7 . Dessa forma, ao serem consumidas, podem proporcionar sensação de saciedade por um período de tempo maior. Assim, acredita-se que a substituição de alimentos de alta densidade energética por alimentos de baixa densidade energética, como frutas e hortaliças, poderia, potencialmente, diminuir a energia total consumida ao longo do dia e impactar no balanço energético ${ }^{8}$.

Sabe-se que comportamentos alimentares inadequados e o baixo nível de atividade física tendem a se agrupar de forma sistemática em nível individual. Tais hábitos merecem atenção, pois essa agregação de fatores pode aumentar o risco de desenvolvimento de sobrepeso e obesidade 9 . Sendo assim, deve-se dar atenção ao padrão de agregação e não apenas a presença isolada 10.

Embora um grande número de estudos tenha avaliado os fatores de risco isolados para a obesidade, poucos investigaram a coexistência desses comportamentos, especialmente em adolescentes 11,12. Assim, acredita-se que conhecer os fatores associados à ocorrência simultânea desses comportamentos pode ser um passo importante para prevenir doenças crônicas não transmissíveis (DCNT) relacionadas ao excesso de peso.

Assim, o objetivo deste estudo foi estimar a prevalência da coocorrência de fatores de risco obesogênicos em adolescentes brasileiros e características sociodemográficas e familiares associadas. Tais informações podem auxiliar na compreensão de quais subgrupos de adolescentes são mais susceptíveis a comportamentos passíveis de afetar o estado nutricional, facilitando a adoção de estratégias de intervenção nessa população, a fim de reduzir a obesidade e suas comorbidades.

\section{Métodos}

\section{Amostra e coleta de dados}

O presente trabalho foi realizado com dados da Pesquisa Nacional de Saúde do Escolar (PeNSE), conduzida no ano de 2009 em todas as capitais brasileiras e no Distrito Federal. Trata-se de um inquérito com amostra representativa de estudantes do 9o ano do Ensino Fundamental de escolas públicas e privadas.

A seleção amostral ocorreu em dois estágios: no primeiro, foram sorteadas as escolas (unidades primárias de amostragem), sendo feita por amostragem sistemática, com probabilidade proporcional ao número de escolas nas cidades. No segundo estágio, foi feito o sorteio das turmas (unidades secundárias). Em cada uma das turmas selecionadas, todos os adolescentes responderam ao questionário da pesquisa, eliminando-se, desta maneira, a necessidade de um terceiro estágio de seleção.

A amostra foi calculada para fornecer estimativas de proporções (ou prevalências) das características de interesse, em cada um dos estratos geográficos (as 26 capitais dos estados e o Distrito Federal), com um erro máximo de $3 \%$ e nível de $95 \%$ de confiança.

A PeNSE 2009 avaliou 60.973 adolescentes. Desses, 53.274 responderam a todas as variáveis que compuseram o desfecho, constituindo, desta maneira, a amostra final do estudo.

A coleta de dados da PeNSE foi realizada por meio de microcomputador de mão (personal digital assistant - PDA), que continha um questionário estruturado e autoaplicável dividido em módulos temáticos: características sociodemográficas, alimentação, imagem corporal, atividade física, tabagismo, consumo de álcool e outras drogas, saúde bucal, comportamento sexual, violência, acidentes e segurança 13 . 


\section{Variáveis do estudo}

A variável desfecho (coocorrência de fatores de risco obesogênicos) foi obtida com base no somatório de cinco comportamentos de risco: consumo diário de refrigerantes adoçados com açúcar; consumo diário de guloseimas, tais como doces, balas, chocolates, chicletes, bombons ou pirulitos; consumo insuficiente de frutas ( $<1 \mathrm{vez} / \mathrm{dia})$; consumo insuficiente de hortaliças ( $<1 \mathrm{vez} / \mathrm{dia})$; e atividade física insuficiente (<300 minutos/semana). As respostas obtidas resultaram em valores de 0 a 5 fatores considerados como obesogênicos. Para a análise, a ocorrência desses fatores foi apresentada em quatro categorias $(0,1,2, \geq 3)$.

A estratégia de dicotomização das variáveis de consumo alimentar utilizada neste estudo foi, também, adotada por outros autores a fim de classificar adolescentes como expostos a padrão alimentar inadequado 14,15,16,17. Em relação à atividade física, foram considerados insuficientemente ativos os adolescentes que acumularam menos de 300 minutos de atividade física na semana anterior à aplicação do questionário, considerando tempo e frequência das atividades de deslocamento, lazer (com ou sem orientação profissional) e atividades desenvolvidas nas aulas de educação física da escola 18,19.

A escolha desses fatores é justificada por serem considerados comportamentos não saudáveis e apontados pela Organização Mundial da Saúde e pela Organização Pan-Americana da Saúde como ponto-chave nas estratégias de prevenção da obesidade na infância e na adolescência 20,21.

As variáveis explicativas foram agrupadas em características sociodemográficas e de contexto familiar. Foram avaliados sexo, faixa etária ( $\leq 13,14,15 \mathrm{e} \geq 16$ anos), cor da pele (branca e não branca), tipo de escola (pública e privada), região brasileira (regiões de menor desenvolvimento socioeconômico: Norte e Nordeste; regiões de maior desenvolvimento socioeconômico: Sul, Sudeste e Centro-oeste), escore de bens e serviços (1o tercil, 2 o tercil e 3o tercil) e escolaridade materna (sem instrução ou Ensino Fundamental incompleto, Ensino Fundamental completo ou Ensino Médio incompleto, Ensino Médio completo ou Ensino Superior incompleto e Ensino Superior completo).

O escore de bens e serviços foi definido com base na posse dos seguintes itens: televisão, geladeira, fogão, micro-ondas, máquina de lavar, telefone fixo, telefone celular, aparelho de DVD, computador, automóvel, banheiro dentro da casa e presença de empregada doméstica em cinco dias ou mais na semana. Cada item recebeu um peso equivalente à frequência de posse dos bens ou presença do serviço no domicílio. Os pesos dos respectivos itens foram somados para a obtenção do escore final para cada adolescente e, posteriormente, categorizados em tercis, sendo que o primeiro tercil diz respeito à presença de menor quantidade de bens e serviços no domicílio.

A escolaridade materna foi analisada em categorias: sem instrução ou Ensino Fundamental incompleto; Ensino Fundamental completo ou Ensino Médio incompleto; Ensino Médio completo ou Ensino Superior incompleto e Ensino Superior completo. Dada a alta porcentagem de valores não informados dessa variável, 18,4\% dos valores foi submetido à imputação. Esse processo compreendeu a identificação de variáveis auxiliares que fossem capazes de predizê-las. A imputação de valores faltantes foi realizada estimando-se o valor médio, após a categorização dos dados em 20 grupos formados com base nas variáveis sexo, tipo de escola e região, como mencionado em publicação prévia 22.

As variáveis de contexto familiar incluídas no estudo foram: composição familiar (reside com a mãe e o pai, somente com a mãe, somente com o pai, e não reside com nenhum dos dois), monitoramento parental (conhecimento dos responsáveis sobre o tempo livre: sempre/na maior parte das vezes e nenhuma vez/raramente/às vezes) e realização de refeições principais com a mãe ou o responsável (5 refeições ou mais por semana e menos que 5 refeições por semana).

\section{Análise estatística}

Inicialmente, foi feita a descrição da população de estudo e estimada a prevalência isolada dos fatores de risco obesogênicos utilizados para a construção da variável desfecho.

O diagrama de Venn foi usado para representar graficamente a ocorrência simultânea de consumo diário de refrigerantes, consumo diário de guloseimas, consumo insuficiente de frutas, consumo insuficiente de hortaliças e atividade física insuficiente. Esse método permite a comparação e a visualização do conjunto de dados, com base na interseção de formas gráficas para mostrar a sobreposição dos fatores avaliados 23 . 
Posteriormente, foi estimada a prevalência do número de fatores de risco obesogênicos, segundo características sociodemográficas e familiares. A associação entre as variáveis explicativas e a coocorrência dos fatores de risco foi realizada por meio do teste de qui-quadrado $\left(\chi^{2}\right)$ e regressão logística ordinal, com modelo de odds proporcionais parciais. Esse modelo é aplicado na análise de dados cuja variável resposta é apresentada em categorias com ordenação e a suposição de chances proporcionais não é válida, impossibilitando o uso do modelo de odds proporcionais 24 . Todas as variáveis explicativas que apresentaram valor de $\mathrm{p}<0,25$ na análise bivariada foram consideradas aptas a entrar no modelo multivariado. Para a seleção das variáveis foi adotado o procedimento stepwise-backward e permaneceram no modelo multivariado final apenas as variáveis com valor de $\mathrm{p}<0,05$. Todas as análises foram feitas no software Stata versão 12 (https://www.stata.com), levando-se em conta a complexidade do desenho amostral.

\section{Aspectos éticos}

A PeNSE 2009 foi aprovada pela Comissão Nacional de Ética em Pesquisa (CONEP) sob o registro no 11.537. A participação dos adolescentes foi voluntária e todas as informações, tanto do aluno quanto da escola, foram confidenciais e não identificadas. A base de dados foi fornecida pela Secretaria de Vigilância em Saúde para a realização do trabalho.

\section{Resultados}

Dos 53.274 adolescentes incluídos neste estudo, 52,7\% eram do sexo feminino, a maioria (65,5\%) pertencia à faixa etária de 14 e 15 anos, 60,4\% possuíam cor da pele branca, 80,2\% eram de escolas públicas, $64,7 \%$ residentes nas regiões de maior desenvolvimento econômico do país, $28 \%$ eram filhos de mães sem instrução ou Ensino Fundamental incompleto, 58,3\% residiam com a mãe e o pai, 55,6\% sempre ou na maioria das vezes tinham monitoramento parental e $62,5 \%$ realizavam cinco ou mais refeições por semana com os responsáveis. A prevalência de 0, 1, 2 e 3 ou mais fatores de risco foi observada em 1,9\%, 8,2\%, 27,1\% e 62,8\% dos adolescentes, respectivamente.

Isoladamente, os fatores de risco mais prevalentes foram o consumo insuficiente de frutas $(82,7 \%$; IC95\%: 82,0-83,3) e hortaliças (80,9\%; IC95\%: 80,2-81,5), seguidos pela atividade física insuficiente (57,5\%; IC95\%: 56,7-58,4) e alto consumo de guloseimas (34,9\%; IC95\%: 33,9-35,8) e refrigerantes (21,6\%; IC95\%: 20,8-22,4) (Figura 1).

A Tabela 1 apresenta a prevalência de fatores de risco obesogênicos isolados, segundo características sociodemográficas e familiares. Nota-se que as características sociodemográficas e parentais se associam de forma distinta aos fatores de risco quando avaliados de forma isolada.

A Figura 2 apresenta o conjunto de fatores de risco que tendem a se agrupar. Por meio do diagrama de Venn, observa-se que as combinações mais prevalentes de fatores de risco foi o consumo inadequado de frutas e hortaliças e inatividade física $(27,1 \%)$, o consumo inadequado de frutas e hortaliças $(16,8 \%)$ e o consumo diário de guloseimas $(9,2 \%)$.

A prevalência de estudantes que reportaram de 0 a 3 ou mais fatores de risco obesogênicos, segundo características sociodemográficas e de contexto familiar é mostrada na Tabela 2. Na análise bivariada, todas as variáveis sociodemográficas e de contexto familiar analisadas mantiveram-se associadas à coocorrência de fatores de risco obesogênicos nos adolescentes.

No modelo final ajustado, perderam a associação estatística as seguintes variáveis: cor da pele, tipo de escola, escore de bens e serviços e composição familiar. Constatou-se maior chance de ocorrência de múltiplos fatores entre os adolescentes do sexo feminino, naqueles que tinham menor monitoramento parental e que realizavam o menor número de refeições com os responsáveis. Por outro lado, adolescentes que residiam em regiões mais desenvolvidas do país e aqueles cujas mães possuíam maior escolaridade apresentaram menor chance de coocorrência de fatores de risco obesogênicos. Essas variáveis mantiveram-se associadas nos três modelos de avaliação (modelo 1: que compara nenhum fator de risco obesogênico versus a presença de um ou mais fatores; modelo 2: compara nenhum ou um fator de risco obesogênico versus a presença de dois ou mais fatores; modelo 3: com- 
Figura 1

Prevalência isolada dos fatores de risco obesogênicos em adolescentes brasileiros. Pesquisa Nacional de Saúde do Escolar, $2009(n=53.274)$.

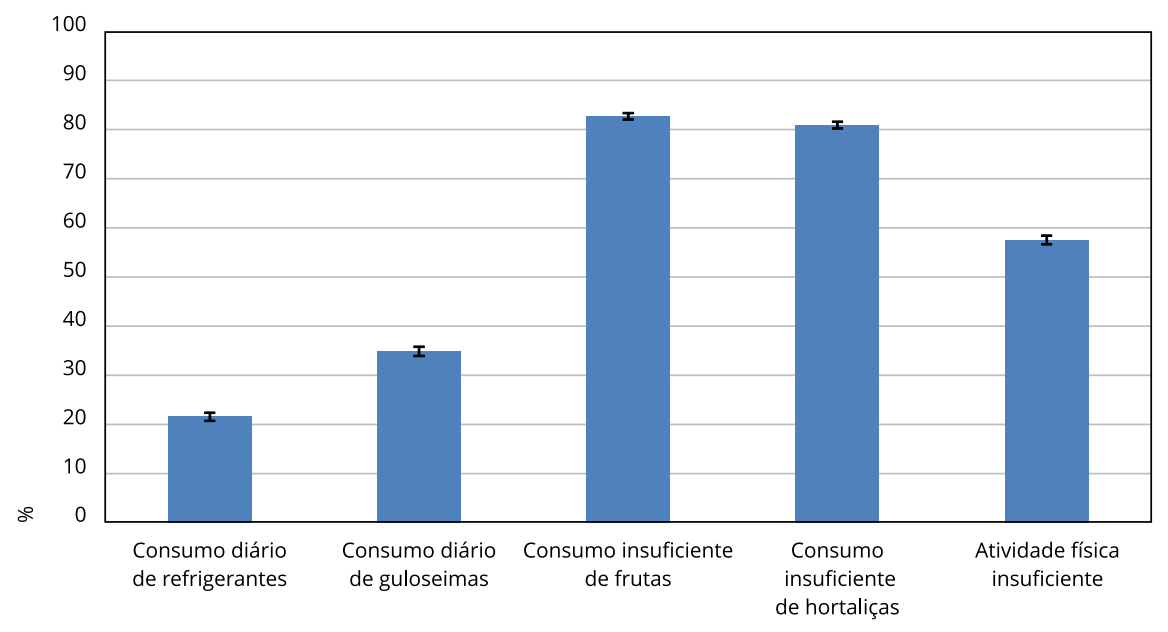

para dois ou menos fatores de risco obesogênicos versus a presença de três ou mais fatores). A faixa etária mais velha foi associada de forma inversa ao desfecho somente no primeiro modelo (Tabela 3).

\section{Discussão}

Múltiplos fatores de risco obesogênicos foram observados em adolescentes residentes nas capitais brasileiras e no Distrito Federal, sendo que a ocorrência simultânea de três ou mais destes fatores foi prevalente em $62,8 \%$ dos adolescentes. Verificou-se maior chance de coocorrência dos fatores de risco obesogênicos nos adolescentes do sexo feminino, naqueles que tinham menor monitoramento parental e que realizavam o menor número de refeições com os responsáveis. Por outro lado, adolescentes mais velhos, que residiam em regiões mais desenvolvidas do país e aqueles que a mãe possuía maior escolaridade apresentaram menor chance de coocorrência desses comportamentos.

A prevalência simultânea de comportamentos de risco à saúde tem sido descrita em diversos estudos. Entretanto, a maioria das pesquisas realizadas com adolescentes tem focado na coocorrência de fatores de risco para o desenvolvimento de DCNT 26,27,28 e poucos têm explorado a agregação de comportamentos obesogênicos, com ênfase na alimentação e na atividade física 11,12.

Elevadas prevalências de múltiplos fatores de risco têm sido observadas em outros estudos. No Reino Unido, Pearson et al. 12 observaram que aproximadamente 70\% dos adolescentes apresentaram dois ou três comportamentos de risco (elevado tempo de tela, baixo consumo de frutas e vegetais, e consumo de lanches de alta densidade energética). Na Austrália, 51\% dos meninos e 43\% das meninas relataram a presença de três ou mais fatores de risco obesogênicos (baixo nível de atividade física, elevado tempo de tela, ingestão insuficiente de frutas e vegetais, alto consumo de refrigerantes e alta ingestão de lanches - fritos e doces) ${ }^{11}$. Entretanto, comparações com esses estudos em termos de grau de coocorrência devem ser interpretadas com cautela devido à diversidade dos métodos utilizados e à variação no conjunto de fatores de risco analisados.

Condizente com outros estudos 28,29, a presente investigação constatou que a presença de maior número de fatores de risco foi mais prevalente nas adolescentes. Relativamente ao comportamento alimentar, o sexo pode influenciar de forma distinta o consumo de alimentos. Em um estudo que 
Tabela 1

Prevalência de fatores de risco obesogênicos isolados, segundo características sociodemográficas e familiares. Pesquisa Nacional de Saúde do Escolar, $2009(n=53.274)$.

\begin{tabular}{|c|c|c|c|c|c|}
\hline Características & $\begin{array}{c}\text { Consumo diário } \\
\text { de refrigerantes } \\
\%(I C 95 \%)\end{array}$ & $\begin{array}{l}\text { Consumo diário } \\
\text { de guloseimas } \\
\%(\text { IC95\%) }\end{array}$ & $\begin{array}{l}\text { Consumo } \\
\text { insuficiente de } \\
\text { frutas } \\
\%(\text { IC95\%) }\end{array}$ & $\begin{array}{l}\text { Consumo } \\
\text { insuficiente de } \\
\text { hortaliças } \\
\%(I C 95 \%)\end{array}$ & $\begin{array}{c}\text { Atividade física } \\
\text { insuficiente } \\
\%(I C 95 \%)\end{array}$ \\
\hline Total da amostra & $21,6(20,7-22,4)$ & $34,9(33,9-35,8)$ & $82,7(82,0-83,3)$ & $80,7(80,2-81,5)$ & $57,5(56,7-58,4)$ \\
\hline Sexo & $p=0,197$ & $p<0,001$ & $p=0,808$ & $p=0,473$ & $p<0,001$ \\
\hline Masculino & $22,04(20,9-23,1)$ & $27,0(25,9-28,1)$ & $82,8(81,9-83,6)$ & $81,1(80,2-82,1)$ & $44,2(43,2-45,3)$ \\
\hline Feminino & $21,5(20,1-22,2)$ & $41,97(40,7-43,2)$ & $82,61(81,7-83,6)$ & $80,6(79,7-81,6)$ & $69,5(68,4-70,6)$ \\
\hline Faixa etária (anos) & $p<0,001$ & $p=0,020$ & $p=0,318$ & $p=0,026$ & $p=0,002$ \\
\hline$\leq 13$ & $19,1(17,6-20,6)$ & $35,3(33,5-37,1)$ & $81,9(80,6-83,3)$ & $79,6(78,4-80,8)$ & $59,7(58,0-61,3)$ \\
\hline 14 & $20,2(19,0-21,3)$ & $34,8(33,5-36,1)$ & $82,6(81,6-83,6)$ & $80,8(79,8-81,8)$ & $57,2(56,0-58,4)$ \\
\hline 15 & $25,9(24,4-27,4)$ & $36,3(34,7-37,9)$ & $83,1(81,6-84,6)$ & $81,6(80,1-83,1)$ & $55,3(53,7-56,9)$ \\
\hline$\geq 16$ & $25,9(24,1-27,7)$ & $31,8(30,0-33,6)$ & $84,1(82,3-86,0)$ & $82,8(81,2-84,4)$ & $57,8(55,7-60,0)$ \\
\hline Cor da pele & $p=0,051$ & $p=0,015$ & $p=0,006$ & $p<0,001$ & $p=0,098$ \\
\hline Branca & $22,5(21,2-23,9)$ & $33,6(32,2-35,1)$ & $81,6(80,6-82,7)$ & $79,3(78,2-80,4)$ & $56,5(55,1-57,9)$ \\
\hline Não branca & $21,0(20,0-21,9)$ & $35,8(34,7-36,9)$ & $83,4(82,6-84,1)$ & $81,9(81,1-82,6)$ & $58,1(56,9-59,3)$ \\
\hline Tipo de escola & $p=0,061$ & $p<0,001$ & $p=0,749$ & $p=0,046$ & $p=0,068$ \\
\hline Pública & $21,1(20,3-21,9)$ & $35,6(34,5-36,7)$ & $82,7(82,0-83,5)$ & $81,1(80,4-81,9)$ & $57,9(57,0-58,9)$ \\
\hline Privada & $23,4(21,1-25,7)$ & $32,0(30,3-33,8)$ & $82,5(81,2-83,8)$ & $79,7(78,4-80,9)$ & $55,8(53,7-57,9)$ \\
\hline Macrorregião & $p<0,001$ & $p<0,001$ & $p<0,001$ & $p<0,001$ & $p<0,001$ \\
\hline Menos desenvolvidas * & $18,2(17,4-19,0)$ & $31,4(30,6-32,3)$ & $87,0(86,5-87,6)$ & $84,7(84,0-85,3)$ & $61,7(60,6-62,7)$ \\
\hline Mais desenvolvidas ** & $23,4(22,2-24,6)$ & $36,8(35,4-38,1)$ & $80,3(79,4-81,2)$ & $78,8(77,8-79,8)$ & $55,3(54,1-56,5)$ \\
\hline Escore de bens e serviços (tercis) & $p<0,001$ & $p<0,001$ & $p<0,001$ & $p<0,001$ & $p=0,079$ \\
\hline 10 & $20,0(18,8-21,3)$ & $31,9(30,6-33,3)$ & $84,0(82,9-85,2)$ & $81,7(80,6-82,7)$ & $57,0(55,6-58,4)$ \\
\hline 20 & $18,6(17,4-19,7)$ & $34,6(33,4-35,8)$ & $84,2(83,4-85,1)$ & $83,3(82,4-84,2)$ & $58,8(57,3-60,4)$ \\
\hline 3으 & $25,2(23,9-26,5)$ & $37,3(35,8-38,9)$ & $80,4(79,0-81,7)$ & $78,2(77,0-79,4)$ & $56,8(55,6-58,1)$ \\
\hline Escolaridade materna & $p=0,006$ & $p=0,037$ & $p<0,001$ & $p<0,001$ & $p<0,001$ \\
\hline $\begin{array}{l}\text { Sem instrução ou Ensino } \\
\text { Fundamental incompleto }\end{array}$ & $19,7(18,5-20,9)$ & $34,9(33,5-36,4)$ & $84,2(82,8-85,7)$ & $83,1(82,2-84,1)$ & $60,5(59,1-62,0)$ \\
\hline $\begin{array}{l}\text { Ensino Fundamental completo ou } \\
\text { Ensino Médio incompleto }\end{array}$ & $22,2(21,1-23,4)$ & $36,1(34,4-37,8)$ & $83,4(82,2-84,5)$ & $82,6(81,2-83,9)$ & $58,5(56,9-60,1)$ \\
\hline $\begin{array}{l}\text { Ensino Médio completo ou Ensino } \\
\text { Superior incompleto }\end{array}$ & $22,3(20,9-23,6)$ & $35,2(33,9-36,5)$ & $82,8(81,7-83,9)$ & $80,1(79,0-81,2)$ & $57,1(55,0-58,5)$ \\
\hline Ensino Superior completo & $22,4(20,5-24,4)$ & $32,3(29,9-34,7)$ & $78,3(76,5-80,2)$ & $74,5(72,8-76,3)$ & $50,7(48,4-53,0)$ \\
\hline Composição familiar & $p=0,523$ & $p<0,001$ & $p=0,048$ & $p=0,002$ & $p<0,001$ \\
\hline Mãe e pai & $21,4(20,5-22,3)$ & $33,7(32,4-34,9)$ & $82,0(81,1-82,8)$ & $79,9(79,1-80,7)$ & $57,1(56,1-58,1)$ \\
\hline Mãe & $21,8(20,5-23,10)$ & $37,0(35,7-38,3)$ & $83,6(82,5-84,7)$ & $82,1(81,1-83,2)$ & $57,7(56,1-59,2)$ \\
\hline Pai & $23,2(20,4-26,0)$ & $32,9(29,8-36,0)$ & $84,4(81,7-87,0)$ & $82,2(79,5-84,8)$ & $55,0(51,9-58,2)$ \\
\hline Nenhum & $20,6(18,1-23,2)$ & $36,7(33,7-39,7)$ & $83,2(80,7-85,7)$ & $82,2(79,8-84,7)$ & $63,6(60,7-66,6)$ \\
\hline Monitoramento parental & $p<0,001$ & $p=0,046$ & $p=0,011$ & $p<0,001$ & $p<0,001$ \\
\hline Sempre/Na maior parte das vezes & $19,1(18,1-20,0)$ & $34,2(32,9-35,4)$ & $81,9(81,1-82,8)$ & $79,0(78,2-79,9)$ & $56,4(55,3-57,5)$ \\
\hline $\begin{array}{l}\text { Nenhuma vez/Raramente/ } \\
\text { Às vezes }\end{array}$ & $24,6(23,4-25,8)$ & $35,9(34,6-37,1)$ & $83,8(82,7-84,8)$ & $83,2(82,2-84,2)$ & $59,1(57,8-60,3)$ \\
\hline $\begin{array}{l}\text { Realização de refeições principais com } \\
\text { a mãe ou responsável (por semana) }\end{array}$ & $p=0,901$ & $p=0,443$ & $p<0,001$ & $p<0,001$ & $p<0,001$ \\
\hline 5 ou mais & $21,6(20,6-22,5)$ & $34,7(33,5-35,9)$ & $80,5(79,6-81,3)$ & $78,1(77,2-79,0)$ & $56,2(55,1-57,2)$ \\
\hline Menos de 5 & $21,6(20,6-22,7)$ & $35,3(34,1-36,5)$ & $86,5(85,5-87,4)$ & $85,7(84,7-86,7)$ & $59,9(58,6-61,2)$ \\
\hline
\end{tabular}

* Norte e Nordeste;

** Centro-oeste, Sudeste e Sul. 


\section{Figura 2}

Coocorrência de fatores de risco obesogênicos (consumo diário de refrigerantes, consumo diário de guloseimas, consumo insuficiente de frutas, consumo insuficiente de hortaliças e inatividade física) em adolescentes brasileiros. Pesquisa Nacional de Saúde do Escolar, 2009 (n = 53.274).



avaliou adolescentes brasileiros, observou-se maior proporção de meninas com ingestão regular de todos os marcadores de dieta não saudável, com exceção do consumo de refrigerantes 30 . De maneira geral, as meninas tendem a adotar práticas alimentares menos saudáveis 31 e serem menos ativas fisicamente 19,32 .

Estudos que avaliaram a agregação de hábitos alimentares e atividade física observaram que adolescentes mais novos eram mais propensos a estar no cluster mais saudável 33,34. No presente trabalho, entretanto, não houve diferença com relação à faixa etária quando se comparou a presença de dois ou mais fatores de risco obesogênicos com um menor número de fatores.

Houve menor chance de exposição a múltiplos fatores de risco nos adolescentes que residiam nas regiões Centro-oeste, Sudeste e Sul, consideradas as mais desenvolvidas do país. Vale ressaltar que as diferenças socioeconômicas e regionais influenciam de forma distinta os fatores de risco obesogênicos quando avaliados de forma isolada. Observou-se maior prevalência de consumo insuficiente de frutas e hortaliças e inatividade física, bem como menor prevalência de consumo diário de refrigerantes nas regiões Norte e Nordeste. Desigualdades regionais têm sido identificadas tanto em relação à disponibilidade de frutas e hortaliças nos domicílios brasileiros 35 quanto no consumo destes alimentos pela população adulta ${ }^{36}$, sendo menor nas regiões Norte e Nordeste do país. Assim, acredita-se que a baixa 
Tabela 2

Proporção de número de fatores de risco obesogênicos em adolescentes brasileiros, segundo características sociodemográficas e de contexto familiar. Pesquisa Nacional de Saúde do Escolar, 2009 ( $\mathrm{n}$ = 53.274).

\begin{tabular}{|c|c|c|c|c|c|}
\hline \multirow[t]{2}{*}{ Características } & \multicolumn{4}{|c|}{$\%$ do número de fatores de risco } & \multirow[t]{2}{*}{ Valor de $\mathrm{p}$} \\
\hline & 0 & 1 & 2 & 3 ou mais & \\
\hline \multicolumn{6}{|l|}{ Sexo } \\
\hline Masculino & 2,58 & 9,76 & 33,73 & 53,93 & $<0.001$ \\
\hline Feminino & 1,41 & 6,75 & 21,07 & 70,76 & \\
\hline Faixa etária (anos) & & & & & 0.004 \\
\hline$\leq 13$ & 1,99 & 8,85 & 26,61 & 62,55 & \\
\hline 14 & 2,01 & 8,47 & 27,73 & 61,80 & \\
\hline 15 & 1,65 & 7,75 & 26,16 & 64,44 & \\
\hline$\geq 16$ & 2,32 & 5,97 & 26,72 & 65,00 & \\
\hline Cor da pele & & & & & $<0.001$ \\
\hline Branca & 2,32 & 9,39 & 27,75 & 60,54 & \\
\hline Não branca & 1,76 & 7,37 & 26,60 & 64,27 & \\
\hline Tipo de escola & & & & & $<0.001$ \\
\hline Pública & 1,74 & 7,96 & 27,03 & 63,27 & \\
\hline Privada & 2,89 & 9,05 & 27,19 & 60,87 & \\
\hline Macrorregião & & & & & $<0.001$ \\
\hline Menos desenvolvidas * & 1,47 & 6,43 & 26,22 & 65,88 & \\
\hline Mais desenvolvidas ** & 2,24 & 9,13 & 27,52 & 61,11 & \\
\hline Escore de bens e serviços (tercis) & & & & & 0.002 \\
\hline $1 \underline{0}$ & 2,18 & 8,02 & 27,09 & 62,71 & \\
\hline 20 & 1,55 & 7,31 & 27,27 & 63,87 & \\
\hline 3o & 2,16 & 9,01 & 26,86 & 61,97 & \\
\hline Escolaridade materna & & & & & $<0.001$ \\
\hline Sem instrução ou Ensino Fundamental incompleto & 1,55 & 6,90 & 26,25 & 65,29 & \\
\hline Ensino Fundamental completo ou Ensino Médio incompleto & 1,47 & 7,39 & 25,70 & 65,44 & \\
\hline Ensino Médio completo ou Ensino Superior incompleto & 1,85 & 8,39 & 28,19 & 61,56 & \\
\hline Ensino Superior completo & 3,94 & 11,84 & 28,96 & 55,25 & \\
\hline Composição familiar & & & & & $<0.001$ \\
\hline Mãe e pai & 2,15 & 8,91 & 27,47 & 61,47 & \\
\hline Mãe & 1,79 & 7,07 & 26,77 & 64,37 & \\
\hline Pai & 1,75 & 8,40 & 27,45 & 62,40 & \\
\hline Nenhum & 1,26 & 6,90 & 24,25 & 67,59 & \\
\hline Monitoramento parental & & & & & $<0.001$ \\
\hline Sempre/Na maior parte das vezes & 2,38 & 9,39 & 28,28 & 59,95 & \\
\hline Nenhuma vez/Raramente/Às vezes & 1,46 & 6,60 & 25,62 & 66,32 & \\
\hline $\begin{array}{l}\text { Realização de refeições principais com a mãe ou responsável } \\
\text { (por semana) }\end{array}$ & & & & & $<0.001$ \\
\hline 5 ou mais & 2,43 & 9,52 & 27,94 & 60,11 & \\
\hline Menos de 5 & 1,22 & 5,84 & 25,51 & 67,43 & \\
\hline
\end{tabular}

* Norte e Nordeste;

** Centro-oeste, Sudeste e Sul. 
Tabela 3

Odds ratio (OR) e intervalo de 95\% de confiança (IC95\%) para 1 ou mais fatores de risco; modelo multivariado das características sociodemográficas e familiares associadas à coocorrência de fatores de risco obesogênicos em adolescentes brasileiros. Pesquisa Nacional de Saúde do Escolar, 2009 $(n=53.274)$.

\begin{tabular}{|c|c|c|c|}
\hline Modelos & OR & Valor de p & IC95\% \\
\hline \multicolumn{4}{|l|}{ 1: Nenhum fator de risco vs. $\geq 1$ fator } \\
\hline \multicolumn{4}{|l|}{ Sexo } \\
\hline Masculino & 1,00 & & \\
\hline Feminino & 1,84 & $<0,001$ & $1,48-2,28$ \\
\hline \multicolumn{4}{|l|}{ Faixa etária (anos) } \\
\hline 13 & 1,00 & & \\
\hline 14 & 0,90 & 0,445 & $0,70-1,17$ \\
\hline 15 & 0,97 & 0,830 & $0,70-1,33$ \\
\hline$\geq 16$ & 0,58 & 0,008 & $0,39-0,87$ \\
\hline \multicolumn{4}{|l|}{ Macrorregião } \\
\hline Menos desenvolvidas * & 1,00 & & \\
\hline Mais desenvolvidas ** & 0,68 & $<0,001$ & $0,56-0,82$ \\
\hline \multicolumn{4}{|l|}{ Escolaridade Materna } \\
\hline Sem instrução ou Ensino Fundamental incompleto & 1,00 & & \\
\hline Ensino Fundamental completo ou Ensino Médio incompleto & 1,13 & 0,540 & $0,77-1,65$ \\
\hline Ensino Médico completo ou Ensino Superior incompleto & 0,94 & 0,746 & $0,65-1,37$ \\
\hline Ensino Superior completo & 0,48 & 0,001 & $0,31-0,73$ \\
\hline \multicolumn{4}{|l|}{ Monitoramento parental } \\
\hline Sempre/Na maior parte das vezes & 1,00 & & \\
\hline Nenhuma vez/Raramente/Às vezes & 1,46 & 0,006 & $1,11-1,91$ \\
\hline \multicolumn{4}{|l|}{ Realização de refeições principais com os responsáveis (por semana) } \\
\hline 5 ou mais & 1,00 & & \\
\hline Menos de 5 & 1,81 & $<0,001$ & $1,44-2,28$ \\
\hline \multicolumn{4}{|l|}{$2: \leq 1$ fator $v s . \geq 2$ fatores } \\
\hline \multicolumn{4}{|l|}{ Sexo } \\
\hline Masculino & 1,00 & & \\
\hline Feminino & 1,58 & $<0,001$ & $1,41-1,77$ \\
\hline \multicolumn{4}{|l|}{ Macrorregião } \\
\hline Menos desenvolvidas * & 1,00 & & \\
\hline Mais desenvolvidas ** & 0,73 & $<0,001$ & $0,66-0,80$ \\
\hline \multicolumn{4}{|l|}{ Escolaridade materna } \\
\hline Sem instrução ou Ensino Fundamental incompleto & 1,00 & & \\
\hline Ensino Fundamental completo ou Ensino Médio incompleto & 1,02 & 0,777 & $0,88-1,19$ \\
\hline Ensino Médico completo ou Ensino Superior incompleto & 0,87 & 0,090 & $0,73-1,02$ \\
\hline Ensino Superior completo & 0,55 & $<0,001$ & $0,45-0,66$ \\
\hline \multicolumn{4}{|l|}{ Monitoramento parental } \\
\hline Sempre/Na maior parte das vezes & 1,00 & & \\
\hline Nenhuma vez/Raramente/Às vezes & 1,38 & $<0,001$ & $1,21-1,57$ \\
\hline \multicolumn{4}{|l|}{ Realização de refeições principais com os responsáveis (por semana) } \\
\hline 5 ou mais & 1,00 & & \\
\hline Menos de 5 & 1,59 & $<0,001$ & $1,38-1,82$ \\
\hline
\end{tabular}

(continua) 
Tabela 3 (continuação)

\begin{tabular}{|c|c|c|c|}
\hline Modelos & OR & Valor de p & IC95\% \\
\hline \multicolumn{4}{|l|}{ 3: $\leq 2$ fatores $v s . \geq 3$ fatores } \\
\hline \multicolumn{4}{|l|}{ Sexo } \\
\hline Masculino & 1,00 & & \\
\hline Feminino & 2,13 & $<0,001$ & $1,99-2,29$ \\
\hline \multicolumn{4}{|l|}{ Macrorregião } \\
\hline Menos desenvolvidas * & 1,00 & & \\
\hline Mais desenvolvidas ** & 0,86 & $<0,001$ & $0,80-0,92$ \\
\hline \multicolumn{4}{|l|}{ Escolaridade materna } \\
\hline Sem instrução ou Ensino Fundamental incompleto & 1,00 & & \\
\hline Ensino Fundamental completo ou Ensino Médio incompleto & 1,13 & 0,260 & $1,11-1,26$ \\
\hline Ensino Médico completo ou Ensino Superior incompleto & 0,92 & 0,080 & $0,84-1,01$ \\
\hline Ensino Superior completo & 0,73 & $<0,001$ & $0,66-0,81$ \\
\hline \multicolumn{4}{|l|}{ Monitoramento parental } \\
\hline Sempre/Na maior parte das vezes & 1,00 & & \\
\hline Nenhuma vez/Raramente/Às vezes & 1,31 & $<0,001$ & $1,22-1,42$ \\
\hline \multicolumn{4}{|l|}{ Realização de refeições principais com os responsáveis (por semana) } \\
\hline 5 ou mais & 1,00 & & \\
\hline Menos de 5 & 1,28 & $<0,001$ & $1,19-1,37$ \\
\hline
\end{tabular}

* Norte e Nordeste;

** Centro-oeste, Sudeste e Sul.

exposição domiciliar às frutas e hortaliças vivenciada pelos adolescentes dessas regiões possa, eventualmente, explicar o baixo consumo desses alimentos e ter impactado nos resultados deste estudo.

Maior escolaridade materna foi associada consistentemente a menores chances de agregação de fatores. Esse resultado é coerente com os encontrados por Ottevaere et al. 34, que observaram que adolescentes cujos pais tinham maior nível educacional eram mais propensos a estar em um cluster mais saudável. Acredita-se que quanto maior o nível de escolaridade, supostamente maior conhecimento a mãe teria sobre os benefícios de se adotarem estilos de vida mais saudáveis e, consequentemente, uma maior possibilidade de proporcionar mais apoio aos filhos 37 . Tem sido observado, também, um maior porcentual de número de fatores de risco comportamentais em jovens que vivem em famílias de baixa renda ou com menor índice de bens no domicílio 11,16,25,28. Informações sobre renda e poder de compra das famílias dos adolescentes não estão disponíveis na PeNSE, no entanto, foi construído um escore considerando-se a presença de bens e serviço no domicílio e este não permaneceu associado no modelo multivariado.

A ausência de monitoramento parental e a realização de menor número de refeições com o responsável permaneceram associadas à presença de múltiplos fatores de risco obesogênicos. Acredita-se que a supervisão dos pais seja considerada importante para prevenir comportamentos de risco em adolescentes com relação ao consumo alimentar e à atividade física, da mesma maneira que exerce efeito protetor sobre outros comportamentos, como o uso de tabaco e álcool e a experimentação de drogas 38 .

A realização de refeições com a família permite momentos de convívio e contato, influenciando a promoção de comportamentos alimentares saudáveis 39 . Esse hábito pode contribuir para o maior consumo de frutas, vegetais e produtos lácteos e menor consumo de bebidas adoçadas com açúcar 40,41. Estudos sugerem, ainda, que a realização de refeições com a família pode exercer efeito protetor na ocorrência de obesidade em adolescentes 42,43. Assim, acredita-se que o apoio e o monitoramento familiar são importantes, tanto quanto a conscientização dos próprios adolescentes para a prevenção de comportamentos de risco obesogênicos. 
Dentre os aspectos que fortalecem os resultados deste estudo inclui a sua representatividade nacional. Entretanto, há que se considerar suas possíveis limitações. Por se tratar de um estudo de delineamento transversal, não há possibilidades de extrapolações sobre fatores causais, mas é possível estabelecer relação de associação. Vale ressaltar, também, que os estudos que avaliaram a coocorrência de fatores de risco para agravos crônicos, como a obesidade, apresentam variações no conjunto de fatores analisados, o que dificulta a comparação entre os resultados. Além disso, a avaliação do consumo alimentar por meio de diferentes métodos e/ou pontos de corte nos estudos incluídos na discussão também pode ser um fator limitante no que diz respeito à comparação dos resultados encontrados. Apesar das limitações mencionadas, os resultados obtidos no presente trabalho são, de maneira geral, consistentes com os encontrados na literatura.

Diante dos achados apresentados, pode-se concluir que há uma proporção considerável de adolescentes brasileiros expostos a fatores de risco obesogênicos de forma simultânea. Ações que estimulem mais envolvimento e supervisão familiar podem promover um impacto positivo para a prevenção da obesidade em adolescentes brasileiros, principalmente em regiões menos desenvolvidas e em lares com mães de menor escolaridade. Além disso, vale ressaltar que torna-se imprescindível a promoção de políticas públicas com foco na elevação dos níveis educacionais da população brasileira e diminuição das desigualdades sociais em níveis regional e nacional.

\section{Colaboradores}

O. C. Chaves, G. Velasquez-Melendez e W. T. Caiaffa contribuíram na análise e interpretação dos dados, elaboração do manuscrito, revisão crítica relevante do conteúdo e aprovação da versão final. D. A. S. Costa e R. G. Andrade contribuíram na análise e interpretação dos dados e aprovação da versão final do manuscrito.

\section{Informações adicionais}

ORCID: Otaviana Cardoso Chaves (0000-00026221-4974); Gustavo Velasquez-Melendez (00000001-8349-5042); Dário Alves da Silva Costa (0000-0002-5959-0370); Roseli Gomes de Andrade (0000-0003-0032-2109); Waleska Teixeira Caiaffa (0000-0001-5043-4980).

\section{Agradecimentos}

Agradecemos à Coordenação de Aperfeiçoamento de Pessoal de Nível Superior (CAPES), à Fundação de Amparo à Pesquisa do Estado de Minas Gerais (FAPEMIG; projeto no PPM00713-16) e ao Ministério da Saúde. 


\section{Referências}

1. Instituto Brasileiro de Geografia e Estatística. Pesquisa de Orçamentos Familiares 20082009. Antropometria e estado nutricional de crianças, adolescentes e adultos no Brasil. Rio de Janeiro: Brasil: Instituto Brasileiro de Geografia e Estatística; 2010.

2. Di Cesare M, Sorić M, Bovet P, Miranda JJ, Bhutta Z, Stevens GA, et al. The epidemiological burden of obesity in childhood: a worldwide epidemic requiring urgent action. BMC Med 2019; 17:212.

3. Martin-Calvo N, Martínez-González MA, Bes-Rastrollo M, Gea A, Ochoa MC, Marti A. Sugar-sweetened carbonated beverage consumption and childhood/adolescent obesity: a case-control study. Public Health Nutr 2014; 17:2185-93.

4. Canella DS, Levy RB, Martins AP, Claro RM, Moubarac J-C, Baraldi LG, et al. Ultra-processed food products and obesity in Brazilian households (2008-2009). PLoS One 2014; 9:e92752.

5. Field AE, Gillman MW, Rosner B, Rockett HR, Colditz GA. Association between fruit and vegetable intake and change in body mass index among a large sample of children and adolescents in the United States. Int J Obes Relat Metab Disord 2003; 27:821-6.

6. Psaltopoulou T, Tzanninis S, Ntanasis-Stathopoulos I, Panotopoulos G, Kostopoulou M, Tzanninis IG, et al. Prevention and treatment of childhood and adolescent obesity: a systematic review of meta-analyses. World J Pediatr 2019; 15:350-81.

7. Boeing H, Bechthold A, Bub A, Ellinger S, Haller D, Kroke A, et al. Critical review: vegetables and fruit in the prevention of chronic diseases. Eur J Nutr 2012; 51:637-63.

8. Guyenet SJ. Impact of whole, fresh fruit consumption on energy intake and adiposity: a systematic review. Front Nutr 2019; 6:66.

9. Leech RM, McNaughton SA, Timperio A. The clustering of diet, physical activity and sedentary behavior in children and adolescents: a review. Int J Behav Nutr Phys Act 2014; 11:4.

10. Visscher TL, Kremers SP. How can we better prevent obesity in children? Curr Obes Rep 2015; 4:371-8.

11. Hardy LL, Grunseit A, Khambalia A, Bell C, Wolfenden L, Milat AJ. Cooccurrence of obesogenic risk factors among adolescents. J Adolesc Health 2012; 51:265-71.

12. Pearson N, Griffiths P, BiddleS JH, Johnston JP, McGeorge S, Haycraft E. Clustering and correlates of screen-time and eating behaviours among young adolescents. BMC Public Health 2017; 17:533.

13. Instituto Brasileiro de Geografia e Estatística. Pesquisa Nacional de Saúde do Escolar. Rio de Janeiro: Instituto Brasileiro de Geografia e Estatística; 2009.
14. Tassitano RM, Barros MVG, Tenório MCM, Bezerra J, Hallal PC. Prevalência e fatores associados ao sobrepeso e à obesidade em adolescentes, estudantes de escolas de Ensino Médio de Pernambuco, Brasil. Cad Saúde Pública 2009; 25:2639-52.

15. Peltzer K, Pengpid S. Fruits and vegetables consumption and associated factors among inschool adolescents in seven African countries. Int J Public Health 2010; 55:669-78.

16. Dumith SC, Muniz LC, Tassitano RM, Hallal PC, Menezes AM Clustering of risk factors for chronic diseases among adolescents from Southern Brazil. Prev Med 2012; 54:393-6.

17. Xavier ICVM, Hardman CM, Andrade MLSS, Barros MVG. Frequência de consumo de frutas, hortaliças e refrigerantes: estudo comparativo entre adolescentes residentes em área urbana e rural. Rev Bras Epidemiol 2014; 17:371-80.

18. World Health Organization. Global recommendations on physical activity for health. Geneva: World Health Organization; 2010.

19. Rezende LFM, Azeredo CM, Canella DS, Claro RM, Castro IRR, Levy RB, et al. Sociodemographic and behavioral factors associated with physical activity in Brazilian adolescents. BMC Public Health 2014; 14:485.

20. WHO Study Group. Diet, nutrition and the prevention of chronic diseases. Geneva: World Health Organization; 2003.

21. Organização Pan-Americana da Saúde. Plano de ação para prevenção da obesidade em crianças e adolescentes. Washington DC: Organização Pan-Americana da Saúde; 2014.

22. Chaves OC, Melendez GV, Costa DAS, Caiaffa WT. Consumo de refrigerantes e índice de massa corporal em adolescentes brasileiros: Pesquisa Nacional de Saúde do Escolar. Rev Bras Epidemiol 2018; 21 Suppl 1:e180010.

23. Heberle H, Meirelles GV, Silva FR, Telles GP, Minghim R. InteractiVenn: a web-based tool for the analysis of sets through Venn diagrams. BMC Bioinformatics 2015; 16:169.

24. Abreu MNS, Siqueira AL, Caiaffa WT. Regressão logística ordinal em estudos epidemiológicos. Rev Saúde Pública 2009; 43; 183-194.

25. Alamian A, Paradis G. Clustering of chronic disease behavioral risk factors in Canadian children and adolescents. Prev Med 2009; 48:493-9.

26. Cureau FV, Duarte P, dos Santos DL, Reichert FF. Clustering of risk factors for noncommunicable diseases in Brazilian adolescents: prevalence and correlates. J Phys Act Health 2014; 11:942-9.

27. Silva KS, Barbosa Filho VC, Del Duca GF, Peres MAA, Mota J, Lopes AS, et al. Gender differences in the clustering patterns of risk behaviours associated with non-communicable diseases in Brazilian adolescentes. Prev Med 2014; 65:77-81. 
28. Nunes HEG, Gonçalves ECA, Vieira JAJ, Silva DAS. Clustering of risk factors for noncommunicable diseases among adolescents from Southern Brazil. PLoS One 2016; 11:e0159037.

29. Plotnikoff RC, Karunamuni N, Spence JC, Storey K, Forbes L, Raine K, et al. Chronic disease-related lifestyle risk factors in a sample of Canadian adolescents. J Adolesc Health 2009; 44:606-9.

30. Azeredo CM, Rezende LFM, Canella DS, Claro RM, Castro IRR, Luiz OC, et al. Dietary intake of Brazilian adolescents. Public Health Nutr 2015; 18:1215-24.

31. Levy RB, Castro IRR, Cardoso LO, Tavares LF, Sardinha LMV, Gomes FS, et al. Consumo e comportamento alimentar entre adolescentes brasileiros: Pesquisa Nacional de Saúde do Escolar (PeNSE), 2009. Ciênc Saúde Colet 2010; 15 Suppl 2:3085-97.

32. Cureau FV, SilvaTLN, BlochKV, Fujimori E, Belfort DR, Carvalho KMB, et al. ERICA: inatividade física no lazer em adolescentes brasileiros. Rev Saúde Pública 2016; 50 Suppl 1:4s.

33. Cameron AJ, Crawford DA, Salmon J, Campbell K, McNaughton SA, Mishra GD, et al. Clustering of obesity-related risk behaviors in children and their mothers. Ann Epidemiol 2011; 21:95-102.

34. Ottevaere C, Huybrechts I, Benser J, De Bourdeaudhuij I, Cuenca-Garcia M, Dallongeville J, et al. Clustering patterns of physical activity, sedentary and dietary behavior among European adolescents: The HELENA study. BMC Public Health 2011; 11:328.

35. Levy-Costa RB, Sichieri R, Pontes NS, Monteiro CA. Household food availability in Brazil: distribution and trends (1974-2003). Rev Saúde Pública 2005; 39:530-40.

36. Jaime PC, Figueiredo ICR, Moura EC, Malta DC. Fatores associados ao consumo de frutas e hortaliças no Brasil, 2006. Rev Saúde Pública 2009; 43 Suppl 2:57-64.
37. Farias Júnior JC, Lopes AS, Mota J, Hallal PC. Prática de atividade física e fatores associados em adolescentes no Nordeste do Brasil. Rev Saúde Pública 2012; 46:505-15.

38. Malta DC, Porto DL, Melo FCM, Monteiro RA, Sardinha LMV, Lessa BH. Família e proteção ao uso de tabaco, álcool e drogas em adolescentes, Pesquisa Nacional de Saúde dos Escolares. Rev Bras Epidemiol 2011; 14 Suppl 1:166-77.

39. Departamento de Atenção Básica, Secretaria de Atenção à Saúde, Ministério da Saúde. Guia alimentar para a população brasileira. Brasília: Ministério da Saúde; 2014.

40. Chan JC, Sobal J. Family meals and body weight. Analysis of multiple family members in family units. Appetite 2011; 57:517-24.

41. Hammons AJ, Fiese BH. Is frequency of shared family meals related to the nutritional health of children and adolescents? Pediatrics 2011; 127:e1565-74.

42. Berge JM, Jin SW, Hannan P, Neumark-Sztainer D. Structural and interpersonal characteristics of family meals: associations with adolescent body mass index and dietary patterns. J Acad Nutr Diet 2013; 113:816-22.

43. Larson N, MacLehose R, Fulkerson JA, Berge JM, Story M, Neumark-Sztainer D. Eating breakfast and dinner together as a family: associations with sociodemographic characteristics and implications for diet quality and weight status. J Acad Nutr Diet 2013; 113:1601-9. 
Abstract

The study aimed to estimate the prevalence of cooccurrence of obesogenic risk factors in Brazilian adolescents and associated sociodemographic and family characteristics. This is a cross-sectional study of data from the Brazilian National School Health Survey, $2009(n=53,274)$. The outcome variable (cooccurrence of obesogenic risk factors) varied from zero to five and was obtained as the sum of the risk behaviors: daily consumption of soft drinks; daily consumption of candy; insufficient consumption of fruits; insufficient consumption of vegetables; and insufficient physical activity. The explanatory variables were sex, age, skin color, region of Brazil, household goods and services score, maternal schooling, type of school, family composition, parental monitoring, and main meals shared with parents or guardians. A Venn diagram was used for exploratory analysis, plus ordinal logistic regression with partial proportional odds model for multivariate analysis. The results showed higher odds of simultaneous occurrence of the factors in adolescent girls, in teens with less parental supervision, and teens who shared fewer meals with their parents or guardians. Meanwhile, adolescents living in more developed regions of the country and those whose mothers had more schooling showed lower odds of cooccurrence of obesogenic risk factors. Actions that encourage greater family involvement and supervision in dietary behavior can have a positive impact on the prevention of obesity in Brazilian adolescents, especially in less developed regions and in homes where the mothers have less schooling.

Obesity; Adolescent; Food Consumption; Risk Factors

\section{Resumen}

El objetivo del estudio fue estimar la prevalencia de la coocurrencia de factores de riesgo obesogénicos en adolescentes brasileños, así como las características sociodemográficas y familiares asociadas. Se trata de un estudio transversal, con datos de la Encuesta Nacional de Salud del Escolar, $2009(n=53.274)$. La variable respuesta (coocurrencia de factores de riesgo obesogénicos) varió de cero a cinco, y se obtuvo a partir del sumatorio de los comportamientos de riesgo: consumo diario de refrescos; consumo diario de golosinas; consumo insuficiente de frutas; consumo insuficiente de hortalizas y actividad física insuficiente. Las variables explicativas fueron: sexo, edad, color de piel, región brasileña, marcador de bienes y servicios del domicilio, escolaridad materna, tipo de escuela, composición familiar, monitoreo parental y realización de comidas principales con los responsables. Se utilizó el diagrama de Venn para un análisis exploratorio y regresión logística ordinal con un modelo de odds proporcionales parciales para el análisis multivariado. Se verificó una mayor oportunidad de ocurrencia simultánea de los factores en adolescentes del sexo femenino, en aquellos que tenían un menor monitoreo parental y que realizaban un menor número de comidas con los responsables. En contraste, adolescentes que residían en regiones más desarrolladas del país, y aquellos cuyas madres poseían una mayor escolaridad, presentaron una menor oportunidad de coocurrencia de factores de riesgo obesogénicos. Acciones que estimulen más implicación y supervisión familiar en el comportamiento alimentario pueden promover un impacto positivo en la prevención de la obesidad en adolescentes brasileños, principalmente en regiones menos desarrolladas y en hogares con madres de menor escolaridad.

Obesidade; Adolescente; Consumo de Alimentos; Factores de Riesgo
Recebido em 27/Jan/2020

Versão final reapresentada em 25/Jun/2020

Aprovado em 10/Ago/2020 